\title{
Validating Measures of Mental Abstraction
}

\author{
Erik Mac Giolla ${ }^{1}$ \\ Timothy J. Luke ${ }^{1}$ \\ Sofia Calderon ${ }^{1}$ \\ Karl Ask ${ }^{1}$ \\ ${ }^{1}$ University of Gothenburg, Department of Psychology \\ 2022-03-01
}

Note: Please do not quote from this preprint. The text may change in future versions.

\section{Author Note}

Corresponding author: Erik Mac Giolla, Department of Psychology, University of Gothenburg, P.O. Box 500, 40530 Gothenburg, Sweden. Email: erik.mac.giolla@psy.gu.se The authors declare no conflict of interest. This project was supported by a grant from the Swedish Research Council awarded to the second author (grant nr. 2019-03037). 


\begin{abstract}
Several dependent variables have been developed to measure mental abstraction. However, the majority of these have not been substantively validated. We tried to rectify this by conducting validation studies of five dependent variables used in past research to measure mental abstraction: the Behavior Identification Form, a segmentation task, a categorization task, and two types of interval estimation tasks. Participants were instructed to think about an object, event, or behavior, in either concrete or abstract terms. We then measured their mental representation of these things using one of the five purported measures of mental abstraction. Only one of the measures - the Behavior Identification Form - registered our strong and direct manipulation of abstraction. We caution against the use of the other four measures and question the conclusions drawn from past research that has used these measures as their primary dependent variable. A greater focus on validating measures of mental abstraction is essential for the continued development of the field.
\end{abstract}

Keywords: validation, mental abstraction, construal level, dependent variables 


\section{Validating Measures of Mental Abstraction}

The human mind can represent things — objects, events, and behaviors — at different levels of abstraction. This ability has interested psychologists for almost a century (Sherif, 1948). The last three decades has seen a resurgence of interest on the topic (Adler \& Sarstedt, 2021; Semin \& Fiedler, 1988; Trope \& Liberman, 2003), and with it the development of an abundance of novel measures of mental abstraction. However, these measures are not without criticism. Foremost among these is that few of these measures have been validated in any substantive manner (Burgoon et al., 2013). Validation of measurements is a cornerstone of scientific research. Without it, we cannot be sure that we are accurately measuring what we claim to measure (Shadish et al., 2002). We address this shortcoming in the literature by attempting to validate several of the extant measures of mental abstraction.

\section{Definitions and Measures of Abstraction}

Exact definitions vary regarding what constitutes an abstract or concrete mental representation (for an overview see Burgoon et al., 2013). However, some common ground in definitions is evident. Most definitions agree that abstraction focuses on characteristics of a thing that are invariant across contexts. Burgoon et al. summarize that abstraction involves "the process of identifying the central characteristics of a thing" (p. 502). Conversely, concretely representing something involves a focus on the particular and varying aspects of a thing. This explains why "canine" is more abstract than "Labrador". The former can describe any dog across any context, whereas the latter only describes one particular breed of dog.

This broad definition of abstraction has further implications that more directly give rise to the many measures of mental abstraction (see Table 1; for a more thorough overview see Burgoon et al., 2013). For instance, if abstraction involves a focus on characteristics of a thing that are invariant across contexts, it follows that abstract representations are more inclusive than concrete representations. This is made clear by the earlier example of canine and Labrador: 
canine can include many more types of dogs than Labrador. That abstraction implies inclusivity is the key idea behind many measures of mental abstraction. This includes segmentation tasks, where more abstract representations of behaviors are indicated by segmenting the behavior into fewer, more inclusive, instances (Wakslak et al., 2006); grouping tasks, where more abstract representations of objects are indicated by grouping the objects into fewer, more inclusive, categories (Liberman et al., 2002); and interval estimation tasks, where more abstract representations of amounts or distances are indicated by broader, more inclusive, interval estimates (Krüger et al., 2014).

A similar argument accounts for the proposal that a description of an action that focuses on why an action is performed is more abstract than a description that focuses on how an action is performed (Vallacher \& Wegner, 1989). The why description is more inclusive and resistant to contextual variations than the how description. This can be illustrated with an example. Consider the action of brushing one's teeth. The reason why we brush our teeth is to maintain good oral hygiene. This why description is inclusive; it can be used to describe many other actions such as flossing, using mouthwash, or going to the dentist. In contrast, describing how we brush our teeth - moving a toothbrush in a circular motion over our teeth-is much less inclusive and cannot be used to describe many other actions. This distinction between a why and how description of an action is the key idea behind the Behavior Identification Form (BIF; Vallacher \& Wegner, 1989), one of the most commonly used measures of mental abstraction (Burgoon et al., 2013).

Another implication from defining abstraction as a focus on invariant characteristics is that abstraction involves a focus on the gist or the whole, as opposed to the particular or constituent parts. This idea is central to the many perceptual measures of abstraction such as the Hierarchical Letters Test (also known as the Navon Letters; Liberman \& Förster, 2009; Navon, 1977) and the Gestalt Completion Test (Ekstrom et al.,1976; Wakslak et al., 2006). In 
the Hierarchical Letters test, large letters are composed of smaller ones (see Figure $1 \mathrm{~A}$ ). It is argued that a more abstract mindset should lead to faster recognition of the larger letter as this is the more holistic interpretation of the image (Liberman \& Förster, 2009). In the gestalt completion test, participants are to identify objects from impoverished images (see Figure 1 B). It is argued that a more abstract mindset should improve performance on the test, as the test requires a holistic interpretation of the stimuli (Wakslak et al., 2006). There are many more perceptual tests of this kind (see Burgoon et al. 2013), however, all rely on similar reasoning to the two described here.

\section{Figure 1}

Example Stimuli from the Hierarchical Letters Test (A) and Gestalt Completion Test (B)

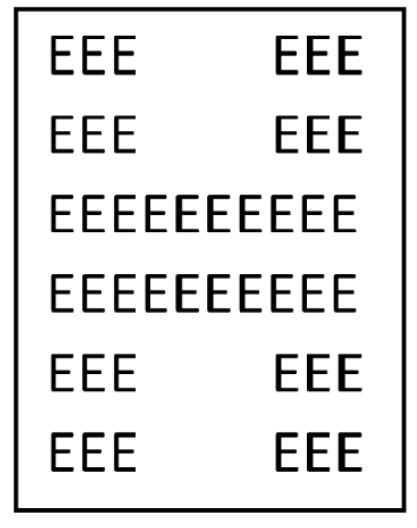

A

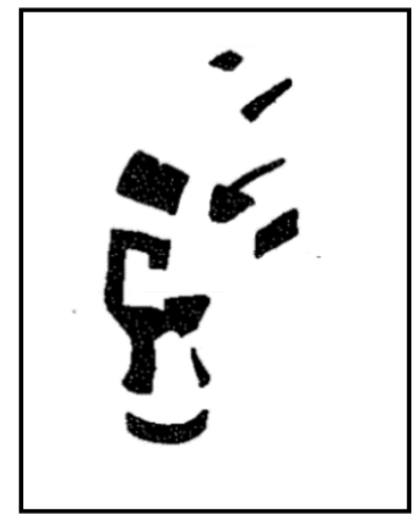

B

A final set of measures of mental abstraction focus on the language people use to describe things. In essence, these measures claim that abstract representations should be described using more abstract language, compared to concrete representations (Semin \& Fiedler, 1991; Bhatia \& Walasek, 2016). There are at least two available ways to measure language abstraction: the linguistic category model (LCM; Semin \& Fiedler, 1991) and folkconceptual dictionaries (Brysbaert et al., 2014). 
Of the two measures, the LCM is more theoretically grounded. LCM argues that verbs and adjectives can be categorized into five distinct categories of increasing abstraction. From least abstract to most abstract these are: descriptive action verbs (e.g., "hit"); interpretive action verbs (e.g., "hurt"); state verbs (e.g., "hate"); adjectives (e.g., "aggressive"); and nouns (e.g., "harasser"; Coenen et al., 2006). To measure how abstract a text is one counts the occurrence of these words and computes an overall abstraction score based on the formula provided by Coenen and colleagues. Traditionally, the LCM was coded manually. However, automated coding procedures have since been developed (Seih et al., 2017).

The folk conceptual dictionary is arguably more straight forward than the LCM. In brief, Brysbaert et al. (2014) had some 4000 participants rate almost 40,000 words on a 5-point scale ranging from 1 (abstract) to 5 (concrete). An average abstractness score was then created for each of the rated words. When calculating the abstractness of a text, one simply averages the abstractness score of each word in the text (Bhatia \& Walasek, 2016).

\section{Table 1}

Overview of abstraction measures categorized by their theoretical justification

\begin{tabular}{ll}
\hline Justification & Measures \\
\hline Abstract representations are more inclusive than & Segmentation Tasks; Grouping tasks; \\
concrete representations & Interval Estimation Tasks
\end{tabular}

Abstract descriptions explain why; concrete Behavior Identification Form
descriptions explain how

Abstraction focuses on the gist, as opposed to Hierarchical letters test; Gestalt completion the particular test 


\author{
Abstract representations are described using \\ LCM; automated text analysis \\ more abstract language
}

\title{
Validating Measures of Mental Abstraction: Importance and Challenges
}

Despite the vast number of measures of mental abstraction, validation studies of these measures are rare (Burgoon et al., 2013). That is, very few studies attempt to demonstrate that these measures actually measure mental abstraction. A lack of validation limits any conclusions based on data from those measures and weakens any theory positing that phenomena observed across several measures relate to the same construct (Cronbach \& Meehl, 1955; Loevinger, 1957; Shadish et al., 2002). For example, if a theory asserted that effects observed across several measures were driven by essentially the same process (e.g., effects of psychological distance on categorization and segmentation tasks occur through the same mechanism), such a claim is jeopardized if there is little or no evidence that the measures function as intended. As such, it is highly important that measures of mental abstraction be validated, given their widespread use.

One approach to validating a new measure is to compare it to an accepted one. Such a comparison allows for both verification and calibration of the new measure. For example, if someone suggests that blood pressure can be measured through blink rate, this novel measure can be compared to a blood pressure monitor-a widely accepted and validated measure of blood pressure. Problematically, there is no such widely accepted and validated measure of mental abstraction. As such, we have no yardstick to assess novel measures, making their validation difficult. This limitation is likely one of the primary reasons for the current state of the literature where validation studies of measures of mental abstraction are virtually nonexistent. 
How then should one validate a measure of mental abstraction if no accepted yardstick exists? In the present research, we used the following logic: A reasonable measure of a construct should always register a strong and direct manipulation of that construct. For instance, both an old-fashioned mercury thermometer and modern resistance thermometer should register a difference in temperature between a cold beer and a hot cup of coffee, even if their precision differs. If a thermometer does not all register this strong and direct manipulation of temperature, its validity is called into question. Following the same logic, we manipulated people's mental representations of things by directly asking them to think of these things in concrete or abstract terms. We then measured their representation of these things with a previously used measure of abstraction. A valid measure of abstraction, should register such a strong and direct manipulation. In the present research, we use this approach to assess the validity of five measures of abstraction: the Behavior Identification Form; a segmentation task; a grouping task; and two forms of interval estimation tasks.

\section{General Method}

All our validation studies followed the same basic approach. We began by identifying studies that used the specific measure of mental abstraction we wanted to validate. In all these original studies, mental abstraction was indirectly manipulated, for instance, by manipulating people's psychological distance from a thing (e.g., Liberman \& Trope, 1998; Wakslak et al., 2006). We used the same basic setup as the original studies, but replaced the indirect manipulation of abstraction, with a direct manipulation. Hence, although we follow the same schematic approach in all of our validation studies, the specific designs vary considerably due to variations in the original studies.

We recruited participants from the crowd-sourced website Prolific. Using websites of this kind is common in the literature (e.g., Krüger et al. 2014; Sánchez et al., 2021). The exact procedures and materials, as well as supplementary analyses and all the data are available on 
the Open Science Framework (https://osf.io/kgrs9/). Due to the large number of studies reported in this paper, descriptions of each study are quite brief. The exact procedures and analysis plans for all the reported studies were preregistered (see https://osf.io/wd437 and https://osf.io/4etu7). Various robustness checks were carried out on the results (e.g., conducting the analyses with or without outliers, or, with or without participants who passed the attention checks). All the analyses reported in the paper were conducted on data from all participants who completed the study (including those who failed attention checks), unless otherwise stated. Furthermore, unless otherwise stated, results did not change in any substantive way during robustness checks.

\section{Sample Size Considerations}

It is important that validation attempts are sufficiently sensitive to detect relevant effects. In their review of manipulation checks in social psychology, Ejelöv and Luke (2020) found that, on average, manipulation checks intended to measure the manipulated construct tended to register effects approximately three times the size of the effect observed on the dependent variable. We use this benchmark to estimate a plausible observable effect for a strong and direct manipulation on a measure of mental abstraction. Soderberg et al. (2015) found that construal level theory studies conducted online (manipulating psychological distance and using a purported measure of abstraction) had an average effect size of $g=.145$. Multiplying this estimate by 3 , we obtain a standardized mean difference of $d=.435$. We consider this a conservative estimate of an effect of a direct manipulation of abstraction on a direct measure of abstraction, given that it is considerably smaller than the average effect of social psychological manipulations on their corresponding manipulation checks (i.e., $d=1.58$; Ejelöv \& Luke, 2020). To achieve $90 \%$ power to observe $d=.435$ with an independent samples $t$-test, a total $N=224$ (group size $n=112$ ) is required $(\alpha=.05)$. Each of the present studies has a sample size greater than $N=224$, so all the present validation attempts had at least $90 \%$ power 
to detect an effect of this size. As such, the present studies are highly sensitive tests of plausible effects.

\section{Study 1: Why and How - The Behavior Identification Form}

The Behavior Identification Form (BIF; Vallacher \& Wegner, 1989) consists of 25 items. Each item contains a description of an action (e.g. "Making a list"). Participants are required to pick one of two options — one abstract and one concrete - that best describes the action. The abstract option describes why the action would be performed (e.g., "getting organized"). The concrete option describes how the action would be performed (e.g., "writing things down"). It is argued that those who think about the actions in more abstract terms will more often choose the abstract options, compared to those who think about the actions in concrete terms (e.g., Fujita et al., 2006; Liberman \& Trope, 1998; Sánchez et al., 2021).

\section{Method}

$N=423$ participants were recruited from Prolific. Participants were randomly assigned to either the abstract condition or concrete condition. The instructions for each condition are presented below.

Abstract condition: We have listed different activities below. Please imagine engaging in each one of them. As you do, please imagine each one in an abstract way. By imagining it in an abstract way, we mean thinking about its purpose and overall significance. In other words, focus on the big picture rather than the specific details.

Concrete condition: We have listed different activities below. Please imagine engaging in each one of them. As you do, please imagine each one in a concrete way. By imagining it in a concrete way, we mean thinking about how it would be to physically do it. In other words, focus on the specific details rather than the big picture.

All participants then received the following instructions: 
Choose one of the two alternative descriptions of the activity that fits best with how you would describe the action. There is no right or wrong answer.

Following this, participants filled out the BIF. To further strengthen the experimental manipulation, before each item participants were instructed to imagine performing the action in either an abstract or concrete way. For example, "Imagine, in [an abstract]/[a concrete] way, yourself making a list." We predicted that those in the abstract condition would score higher on the BIF.

\section{Results}

Consistent with predictions, participants' BIF scores in the abstract condition $(M=$ $16.52, S D=7.03)$ were greater than those in the concrete condition $(M=6.78, S D=6.77), d=$ $1.42,95 \%$ CI $[1.20,1.63]$.

\section{Study 2: Abstraction Measures Based on Inclusivity}

In Study 2, we attempt to validate four different measures of abstraction that all build on the basic idea that abstract representations of things should be more inclusive than concrete representations. The four measures we examined were a segmentation task, a categorization task, and two types of interval estimation tasks (length estimates and quantity estimates). Study 1, and studies $2 \mathrm{a}$, and $2 \mathrm{~b}$ were conducted simultaneously, and participants were randomly assigned to take part in one of those three studies. Studies $2 \mathrm{c}, 2 \mathrm{~d}$, and $2 \mathrm{e}$ were conducted separately.

\section{Study 2a: Segmentation Tasks}

When measuring abstraction using a segmentation task, participants are required to watch a video of some behavior. They are then instructed to segment the video into "meaningful action units" by pressing a keyboard key (e.g., the spacebar) every time they believe a meaningful action ends and another begins. Participants who segment the video into fewer, 
more inclusive parts are thought to have a more abstract representation of the behavior depicted in the video (e.g., Henderson et al., 2006; Wakslak et al., 2006).

Method. $N=410$ participants were recruited from Prolific. We used the same basic setup as Henderson et al. (2006). Participants were told to imagine that they had been hired for a new position in a film production company and that as part of their job they were required to watch a rough sketch of an event that took place on a camping trip for teenagers. The video depicted geometric shapes moving in a way that can readily be understood narratively (adapted from Heider \& Simmel, 1944). Half of the participants were randomly assigned to the abstract condition and the other half to the concrete condition.

We instructed those in the abstract condition to:

Please imagine the camping trip in an abstract way. By imagining it in an abstract way, we mean thinking about its purpose and overall significance. In other words, focus on the big picture rather than the specific details.

We instructed those in the concrete condition to:

Please imagine the camping trip in a concrete way. By imagining it in a concrete way, we mean thinking about how it would be to physically do it. In other words, focus on the specific details rather than the big picture.

All participants then received the same instructions to segment the video by pressing the spacebar, whenever they believed a meaningful action unit ended and another began. Before watching the video, participants were again reminded to imagine the activities in the video in either an abstract or concrete way. We predicted that those in the abstract condition would segment the video into fewer parts compared to the participants in the concrete condition.

Results. Against predictions, there was no significant difference in segmentation (i.e., spacebar presses) between participants in the abstract condition $(M=11.30, S D=9.66)$ and those in the concrete condition $(M=11.95, S D=9.85), d=0.066,95 \% \mathrm{CI}[-0.123,0.262]$. 


\section{Study 2b: Categorization Tasks}

When measuring abstraction with a categorization task, participants are presented with a group of items of relevance to some particular task or event. It is thought that participants who represent the event in more abstract terms should group the items into fewer, more inclusive, categories (e.g., Liberman et al., 2002).

Method. $N=355$ participants were recruited from Prolific. We used the same basic setup as Liberman et al. (2002). Participants were presented with three hypothetical scenarios (a camping trip; a yard sale; and moving house) and asked to categorize objects related to each scenario. For example, for the yard sale participants were given a list of 39 items that could plausibly be sold at a yard sale (e.g., ceramic figurines, dolls, lamps). Half of the participants were instructed to think of the three scenarios in an abstract way, the other half were instructed to think of the three scenarios in a concrete way. As an example, we provide the instructions for the yard sale.

Abstract condition: Please imagine having a yard sale. We would like you to imagine this event in an abstract way. By imagining it in an abstract way, we mean thinking about its purpose and overall significance. In other words, focus on the big picture rather than the specific details.

Concrete condition: Please imagine having a yard sale. We would like you to imagine this event in a concrete way. By imagining it in a concrete way, we mean thinking about how it would be to physically do it. In other words, focus on the specific details rather than the big picture.

Participants were then instructed to look at the list of items and place them into groups by dragging and dropping items that belong together into the same box. We predicted that those 
in the abstract condition would categorize the items into fewer groups compared to those in the concrete condition.

Results. Data were analyzed with participants' responses to each scenario as the unit of analysis ( $N=1,062$ observations). Against predictions, there was no significant difference in the number of categories generated between the abstract condition $(M=5.49, S D=2.00)$ and the concrete condition $(M=5.68, S D=2.44), d=0.087,95 \%$ CI $[-0.035,0.208]$.

\section{Study 2c: Interval Estimation Tasks (Length)}

When measuring abstraction using a length interval estimates, participants are asked to judge the minimum and maximum length of an object (e.g., a bridge). It is argued that more abstract representations of the object should produce wider, more inclusive, intervals (Krüger et al., 2014).

Method. $N=245$ participants were recruited from Prolific. We excluded data from six participants who provided invalid responses (they provided max estimates smaller than the min estimates; final sample $N=239$ ). We followed the basic setup of Krüger et al. (2014). Participants were presented with 12 pictures of bridges. They were required to make interval estimates of the length of each bridge, by suggesting a minimum and maximum length. The interval estimate for each bridge was calculated by subtracting the minimum estimate from the maximum estimate. Participants were randomly allocated to the abstract or concrete condition.

All participants read the following text describing basic differences between concrete or abstract representations:

When thinking of objects in concrete terms we focus on the details of their physical properties. In other words, we focus on the specific details rather than the big picture.

In contrast, when thinking of objects in abstract terms, we focus on their purpose and what they can be used for. In other words, we focus on the big picture rather than the specific details. 
Depending on the experimental condition, participants were then told they were to imagine the bridges in either abstract or concrete terms. Participants were then randomly presented with the 12 images of bridges. Under each image, participants were reminded to think of the bridge in either concrete or abstract terms.

Abstract condition. When thinking about this bridge, we would like you to imagine it in an abstract way. By imagining it in an abstract way, we mean thinking about its purpose and what it could be used for. In other words, focus on the big picture rather than the specific details.

Concrete condition. When thinking about this bridge, we would like you to imagine it in a concrete way. By imagining it in a concrete way, we mean thinking about the details of its physical properties. In other words, focus on the specific details rather than the big picture.

We predicted that those in the abstract condition would provide wider interval estimates compared to participants in the concrete condition. After completing the estimation tasks, participants filled out the BIF (Vallacher \& Wegner, 1989). This was to investigate potential spillover effects of an abstract or concrete mindset on a different measure of abstraction. In accordance with Krüger et al. (2014) it was predicted that those in the abstract condition would score higher on the BIF, indicating a more abstract mindset. For more details on the BIF, see Study 1 .

Results. Consistent with the approach by Krüger et al (2014), we calculated z-scores for the interval estimates for each bridge and then calculated an average z-score for each participant. The primary dependent variable was these mean standardized interval estimates. Against predictions, there was no significant difference in the mean standardized interval estimates between the abstract condition $(M=0.19, S D=0.93)$ and the concrete condition $(M$ $=0.10, S D=0.90), d=0.097,95 \% \mathrm{CI}[-0.158,0.352]$. Additionally, there was no significant 
difference in BIF scores between the abstract condition $(M=13.93, S D=5.48)$ and the concrete condition $(M=13.24, S D=4.74), d=0.134,95 \% \mathrm{CI}[-0.118,0.386]$.

\section{Study 2d: Interval Estimation Tasks (Quantity)}

When measuring abstraction using quantity interval estimates, participants are asked to judge the minimum and maximum number of items in a container (e.g., berries in a bowl). It is argued that more abstract representations of the items should produce wider, more inclusive, interval estimates (Krüger et al. 2014).

Method. $N=244$ participants were recruited from Prolific. We excluded the data from two participants who provided invalid responses (they provided max estimates smaller than the min estimates; final sample $N=242$ ). We followed the basic setup of Krüger et al. (2014). Participants were presented with 10 pictures of containers holding varying amounts of fruits, vegetables, or nuts. They were required to make interval estimates of the number of items in each container, by suggesting a minimum and maximum number. The interval estimate for each container was calculated by subtracting the minimum estimate from the maximum estimate. Participants were randomly allocated to the abstract or concrete condition.

All participants read the same text reported in Study 1c, describing basic differences between concrete or abstract representations. Participants were then told they were to imagine the image in either abstract or concrete terms. Participants were then randomly presented with the 10 images. Under each image, participants were reminded to think of the image in either abstract or concrete terms. Furthermore, in the abstract condition, the content of the container was described using a more abstract term (e.g., fruit) than in the concrete condition (e.g., blueberries

Abstract condition. When thinking about this bowl of fruit, we would like you to imagine it in an abstract way. By imagining it in an abstract way, we mean thinking about its purpose 
and what it could be used for. In other words, focus on the big picture rather than the specific details.

Concrete condition. When thinking about this bowl of blueberries, we would like you to imagine it in a concrete way. By imagining it in a concrete way, we mean thinking about the details of its physical properties. In other words, focus on the specific details rather than the big picture.

We predicted that those in the abstract condition would provide wider interval estimates compared to participants in the concrete condition. After completing the estimation tasks, participants filled out the BIF (Vallacher \& Wegner, 1989) in order to investigate potential spillover effects.

Results. Consistent with the approach by Krüger et al. (2014), we calculated z-scores for the interval estimates for each image and then calculated an average z-score for each participant. The primary dependent variable was these mean standardized interval estimates. Against predictions, there was no significant difference in the mean standardized interval estimates between the abstract condition $(M=0.10, S D=0.78)$ and the concrete condition $(M$ $=0.02, S D=0.76), d=0.100,95 \% \mathrm{CI}[-0.154,0.353]$. Additionally, there was no significant difference in BIF scores between the abstract condition $(M=12.50, S D=5.22)$ and the concrete condition $(M=13.34, S D=5.27), d=-0.159,95 \%$ CI $[-0.412,0.093]$.

\section{Study 2e: Close replication of Krüger et al. (2014, Study. 4)}

This study was a close replication of Krüger et al. (2014, Study 4). In this experiment, the authors aimed to more directly manipulate construal level (rather than psychological distance) by changing the way in which the objects depicted in the images were described. Specifically, they described the objects in either high-level terms (i.e., the category of object to which they belong) or low-level terms (i.e., as an exemplar of a category). Thus, Krüger et al. (2014, Study 4) served a purpose similar to the other present validation studies: to 
demonstrate that interval estimates are responsive to a relatively direct manipulation of mental abstraction. As such, we believed it was worthwhile to conduct a close replication of this experiment. If we failed to observe an effect using the manipulation we used with the other validation studies, but successfully observed an effect with the original manipulation used by Krüger et al. (2014), such a result would suggest that our validation manipulation may have been ineffective. However, if we failed to observe an effect with the original manipulation, such a result would suggest that either the original manipulation or the dependent measure were ineffective. Krüger et al. (2014, Study 4) observed an effect of their manipulation on mean standardized interval estimates, $d=0.57,95 \% \mathrm{CI}[0.07,1.06]$ and on the $\mathrm{BIF}, d=0.57,95 \% \mathrm{CI}$ $[0.07,1.06]$.

Method. $N=248$ participants were recruited from Prolific. We excluded data from one participant who provided invalid responses (they provided max estimates smaller than the min estimates; final sample $N=247$ ). Participants made the same quantity estimates of the same stimuli as in Study 2d. The only difference between this study and Study $2 \mathrm{~d}$ was the experimental manipulation. Again, participants were randomly assigned to an abstract or concrete condition. However, rather than directly manipulating abstraction, as was done in Study $2 \mathrm{~d}$, the contents of the containers were described using terms that were either more abstract (fruit, nuts, vegetables) or concrete (blueberries, pistachios, cucumbers). We predicted that those in the abstract condition would provide wider interval estimates compared to participants in the concrete condition. After completing the estimation tasks participants filled out the BIF, in order to investigate potential spillover.

We tried to keep the current study as similar as possible to Krüger et al. (2014, Study 4). The primary differences were: (1) we used a new set of images as we did not have access to the original ones; (2) we recruited participants from Prolific rather than Amazon Mechanical Turk; and (3) we used the original BIF scale, rather than the context specific one created by 
Krüger et al. (2014), as we could not obtain the items of their amended BIF scale. We asked one of the original authors to review our material for any obvious deviations from the original study. The author did not note any issues with the material, however, they suggested using a slider with a constrained upper and lower bound estimate, in order to avoid outliers (T. Krüger, personal communication, January 2022). In order to keep our results as comparable as possible to the original study we opted against this suggestion.

Results. Consistent with the approach by Krüger et al. (2014), we calculated z-scores for the interval estimates for each image and then calculated an average z-score for each participant. The primary dependent variable was these mean standardized interval estimates. Against predictions, there was no significant difference in the mean standardized interval estimates between the abstract condition $(M=0.05, S D=0.79)$ and the concrete condition $(M$ $=0.11, S D=0.84), d=-0.081,95 \%$ CI $[-0.332,0.170]$. Additionally, there was no significant difference in BIF scores between the abstract condition $(M=11.97, S D=5.86)$ and the concrete condition $(M=12.15, S D=5.68), d=-0.031,95 \% \mathrm{CI}[-0.281,0.219]$. In short, we failed to replicate the results of Krüger et al. (2014, Study 4). Our observed effects were nonsignificant, in the opposite direction to the hypothesis, and their 95\% CIs excluded the original effect estimates.

\section{Summary of Results}

With the notable exception of the BIF, the $95 \%$ confidence intervals for all estimated effects for all purported measures of mental abstraction included 0 and excluded our conservative estimate of the expected effect (i.e., $d=.435$; see Figure 2). These results suggest that if categorization, segmentation, or interval estimation, measure mental abstraction at all, they are highly insensitive measures of abstraction. Moreover, in the tests of the interval estimation task, we failed to observe spillover effects on the BIF. It is possible that the BIF is 
relatively insensitive to general shifts in mindset and that its primary utility is detecting changes in mental abstraction directly pertaining to the items on the measure.

\section{Figure 2}

Overview of Effect Sizes

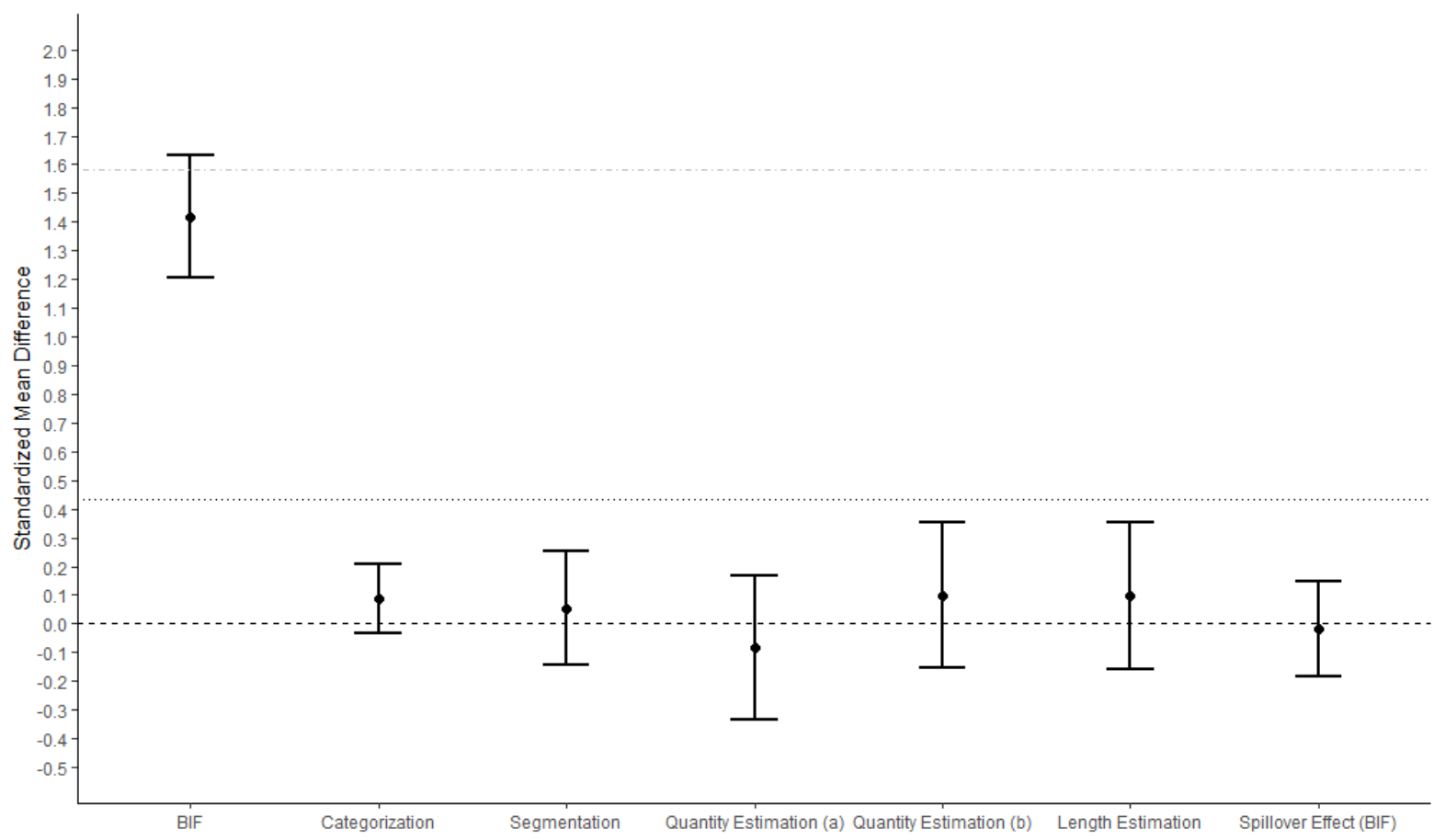

Note. Dotted line refers to a conservative expectation of an effect of a direct manipulation of abstraction ( $d=0.435$; see section on Sample Size Considerations). Dashdotted line refers to the average effect size for manipulation checks reported in social psychology $(d=1.58$, Ejelöv \& Luke, 2020). Error bars represent the $95 \%$ confidence intervals. The spillover effect is the weighted average of the three validation studies that tested this. Effect sizes are calculated such that positive values correspond to effects in the expected direction.

\section{Discussion}

The aim of this study was to validate common measures of mental abstraction. We did this by directly asking participants to construe things in either abstract or concrete terms and 
then measured their representations of these things. We assessed five purported measures of abstraction. Of the five measures, only one-the Behavior Identification Form (BIF)registered our strong and direct manipulation of abstraction. Put differently, we were able to validate only one of the five measures. Below, we discuss reasons for the poor performance of the other four measures, before highlighting the pros and cons of the BIF.

Based on the current study, neither categorization, segmentation, or interval estimation appear to measure mental abstraction. This result is at odds with previous research that has used these measures to demonstrate differences in mental abstraction. For instance, the measures have been used in several studies inspired by Construal Level Theory (Liberman \& Trope, 2010), where mental abstraction is typically indirectly manipulated by manipulating participants' psychological distance to objects, people, and events (Krüger et al. 2014; Liberman et al, 2002; Wakslak et al., 2006).

One potential explanation for the discrepancy between past research and the current findings is that our direct manipulation of abstraction was ineffective. Perhaps the definition of abstraction we used in our manipulation was inappropriate or too vague. Alternatively, perhaps direct manipulations of abstraction cause reactance in participants, leading to an ironic reduction in the very behavior the manipulation is intended increase (Brehm, 1966). Speaking against both of these explanations is that our direct manipulation of abstraction worked as expected for one of the measures, the BIF. Furthermore, this produced an effect $(d=1.42)$ comparable to the average effect of manipulation checks in social psychology $(d=1.58$; Ejelöv \& Luke, 2020). Moreover, if our manipulation was ineffective, the effect on the BIF would still demand an explanation, and the existence of this effect would carry problematic implications for the validity of the BIF. If we assume the manipulation did not function as intended, because participants' scores on the BIF were clearly influenced by the manipulation, we would then have to conclude that the BIF is highly responsive to face-valid but spurious stimuli. This 
conclusion would problematize existing conclusions in the literature that rely on the BIF, especially if there is a possibility of influence by constructs other than mental abstraction (for examination of a similar problem, see Luke et al., 2022).

However, if our manipulation of mental abstraction was indeed effective, the current findings imply that categorization, segmentation, interval estimations of quantity, and interval estimations of length simply do not reliably measure differences between concrete and abstract representations of things, at least in their present configurations. Given that previous research that uses these measures sometimes demonstrates an experimental effect on abstraction, this interpretation implies that previous findings may have been false positives or may be caused by constructs other than abstraction. The possibility that the literature contains many false positives, though contentious, aligns with difficulties in replicating previous findings using these measures, both in the current work (see Study 2e) and previous work (Calderon et al., 2020). That previous findings may be false positives is also in accordance with the extreme publication bias evident in meta-analyses of research fields where these measures are used (Maier et al., 2022). However, it is also possible that many effects are true positives, in that they correspond to authentically replicable phenomena, but their theoretical explanation may be different from the interpretations offered by those studies' authors. That is, their internal validity may be intact, even if their construct validity is in peril (Shadish et al., 2002). Both of these possibilities are highly problematic for the state of the literature.

The BIF was the only measure that successfully registered our direct manipulation of abstraction. The BIF has many desirable attributes. For example, its constituent items have a clear correspondence to the construct of abstraction, specifically the distinction between why and how. However, the BIF is not without its limitations. Previous research has used the BIF as a measure of more general shifts of mindset. For instance, when an abstract or concrete mindset is subtly primed (Krüger et al. 2014; Wakslak et al., 2006). Problematically, in three 
studies, we did not observe any clear spillover effects of mindset on the BIF (including a replication whose original previously observed spillover effects). Thus, the BIF may only register manipulations in mindset targeted directly at the 25 -items included in the measure. Unfortunately, this means that the type of research that this measure can be used for may be quite limited in scope.

\section{Conclusions}

Most measures of mental abstraction have not been sufficiently validated (Burgoon et al., 2013). This reflects a broader issue in psychological research: the rush to test hypotheses before the constructs and measures underpinning the hypotheses have been sufficiently explored (Scheel et al., 2021). Our results highlight the consequences of ignoring this vital part of scientific research. Simply put, we were unable to validate four out of the five measures of abstraction we examined. These results provide a warning to researchers who wish to use these four measures in future studies and calls into question the conclusions drawn by research that have availed of these measures in the past. Continued work on the validation of measures of mental abstraction is essential for progress in the field. 


\section{References}

Adler, S., \& Sarstedt, M. (2021). Mapping the jungle: A bibliometric analysis of research into construal level theory. Psychology \& Marketing, 38(9), 1367-1383. https://doi.org/10.1002/mar.21537

Bhatia, S., \& Walasek, L. (2016). Event construal and temporal distance in natural language. Cognition, 152, 1-8. https://doi.org/10.1016/j.cognition.2016.03.011

Brehm, J. W. (1966). A theory of psychological reactance, New York: Academic Press.

Brysbaert, M., Warriner, A. B., \& Kuperman, V. (2014). Concreteness ratings for 40 thousand generally known English word lemmas. Behavior Research Methods, 46(3), 904-911. https://doi.org/10.3758/s13428-013-0403-5

Burgoon, E. M., Henderson, M. D., \& Markman, A. B. (2013). There are many ways to see the forest for the trees: A tour guide for abstraction. Perspectives on Psychological Science, 8(5), 501-520.

Calderon, S., Mac Giolla, E., Ask, K., \& Granhag, P. A. (2020). Subjective Likelihood and the Construal Level of Future Events: A Replication Study of Wakslak, Trope, Liberman, and Alony (2006). Journal of Personality and Social Psychology, 119, e27-e37. https://doi.org/10.1037/pspa0000214

Coenen, L. H., Hedebouw, L., \& Semin, G. R. (2006). The linguistic category model (LCM) manual. Unpublished manuscript.

Cronbach, L. J., \& Meehl, P. E. (1955). Construct validity in psychological tests. Psychological Bulletin, 52(4), 281-302.

Ejelöv, E., \& Luke, T. J. (2020). "Rarely safe to assume": Evaluating the use and interpretation of manipulation checks in experimental social psychology. Journal of Experimental Social Psychology, 87, 103937. https://doi.org/10.1016/j.jesp.2019.103937 
Ekstrom, R. B., French, J. W., Harman, H. H., \& Dermen, D. (1976). Kit of factor-referenced cognitive tests. Princeton, NJ: Educational Testing Service.

Fujita, K., Henderson, M., Eng, J., Trope, Y., \& Liberman, N. (2006). Spatial distance and mental construal of social events. Psychological Science, 14, 278-282. https://doi.org/10.1111/j.1467-9280.2006.01698.x

Henderson, M. D., Fujita, K., Trope, Y., \& Liberman, N. (2006). Transcending the "here": the effect of spatial distance on social judgment. Journal of Personality and Social Psychology, 91(5), 845. https://doi.org/10.1037/0022-3514.91.5.845

Krüger, T., Fiedler, K., Koch, A. S., \& Alves, H. (2014). Response category width as a psychophysical manifestation of construal level and distance. Personality and Social Psychology Bulletin, 40(4), 501-512.

Liberman, N., \& Förster, J. (2009). The effect of psychological distance on perceptual level of construal. Cognitive Science, 33, 1330-1341.

Liberman, N., \& Trope, Y. (1998). The role of feasibility and desirability considerations in near and distant future decisions: A test of temporal construal theory. Journal of personality and social psychology, 75(1), 5. https://doi.org/10.1037/0022-3514.75.1.5

Liberman, N., Sagristano, M. D., \& Trope, Y. (2002). The effect of temporal distance on level of mental construal. Journal of Experimental Social Psychology, 38(6), 523-534. https://doi.org/10.1016/S0022-1031(02)00535-8

Loevinger, J. (1957). Objective Tests as Instruments of Psychological Theory. Psychological Reports, 3(3), 635-694. https://doi.org/10.2466/pr0.1957.3.3.635

Luke, T. J., Ask, K., Magnusson, E., Calderon, S., \& Mac Giolla, E. (2022). Revisiting the Relationship Between Social Distance and Communication Preferences: Replications and Reinterpretation of Amit et al. (2013, Experiment 2). https://doi.org/10.31234/osf.io/qjw7g 
Navon, D. (1977). Forest before trees: The precedence of global features in visual perception. Cognitive Psychology, 9, 353-383.

Sánchez, A. M., Coleman, C. W., \& Ledgerwood, A. (2021). Does temporal distance influence abstraction? A large pre-registered experiment. Social cognition, 39(3), 352365. https://doi.org/10.1521/soco.2021.39.3.352

Scheel, A. M., Tiokhin, L., Isager, P. M., \& Lakens, D. (2021). Why hypothesis testers should spend less time testing hypotheses. Perspectives on Psychological Science, 16(4), 744-755.

Seih, Y.-T., Beier, S., \& Pennebaker, J. W. (2017). Development and examination of the linguistic category model in a computerized text analysis method. Journal of Language and Social Psychology, 36(3), 343-355. https://doi.org/10.1177/0261927X16657855

Semin, G. R., \& Fiedler, K. (1991). The linguistic category model, its bases, applications and range. European Review of Social Psychology, 2, 1-30.

Shadish, W., T. Campbell and D. Cook, (2002). Experimental and quasi-experimental designs for generalized causal inference. Houghton and Mifflin.

Sherif, M. (1948). An Outline of Social Psychology. New York: Harper \& Row.

Soderberg, C. K., Callahan, S. P., Kochersberger, A. O., Amit, E., \& Ledgerwood, A. (2015). The effects of psychological distance on abstraction: Two meta-analyses. Psychological Bulletin, 141(3), 525. https://doi.org/10.1037/bul0000005

Trope, Y., \& Liberman, N. (2003). Temporal construal. Psychological Review, 110, 403-421. Vallacher, R. R., \& Wegner, D. M. (1989). Levels of personal agency: Individual variation in action identification. Journal of Personality and Social psychology, 57(4), 660-671.

Wakslak, C. J., Trope, Y., Liberman, N., \& Alony, R. (2006). Seeing the forest when entry is unlikely: probability and the mental representation of events. Journal of Experimental 
Psychology: General, 135(4), 641. https://psycnet.apa.org/doi/10.1037/0096-

$\underline{3445.135 .4 .641}$ 